\title{
Article
}

\section{Is metacognition a causal moderator of the relationship between catastrophic misinterpretation and health anxiety? A prospective study}

Bailey, Robin and Wells, Adrian

Available at http://clok.uclan.ac.uk/13766/

Bailey, Robin and Wells, Adrian (2016) Is metacognition a causal moderator of the relationship between catastrophic misinterpretation and health anxiety? A prospective study. Behaviour Research and Therapy, 78 . pp. 43-50. ISSN 00057967

It is advisable to refer to the publisher's version if you intend to cite from the work. http://dx.doi.org/10.1016/j.brat.2016.01.002

For more information about UCLan's research in this area go to http://www.uclan.ac.uk/researchgroups/ and search for <name of research Group>.

For information about Research generally at UCLan please go to http://www.uclan.ac.uk/research/

All outputs in CLoK are protected by Intellectual Property Rights law, including Copyright law. Copyright, IPR and Moral Rights for the works on this site are retained by the individual authors and/or other copyright owners. Terms and conditions for use of this material are defined in the policies page. 


\section{Accepted Manuscript}

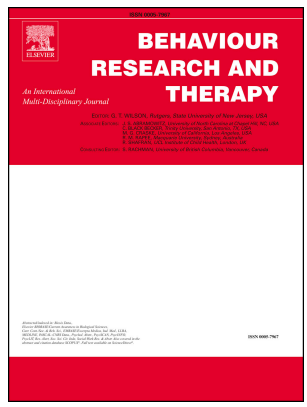

Is Metacognition a Causal Moderator of the Relationship Between Catastrophic Misinterpretation and Health Anxiety? A Prospective Study

Robin Bailey, Adrian Wells

PII: S0005-7967(16)30009-2

DOI: 10.1016/j.brat.2016.01.002

Reference: $\quad$ BRT 2961

To appear in: Behaviour Research and Therapy

Received Date: 17 September 2015

Revised Date: 30 December 2015

Accepted Date: 23 January 2016

Please cite this article as: Bailey, R., Wells, A., Is Metacognition a Causal Moderator of the Relationship Between Catastrophic Misinterpretation and Health Anxiety? A Prospective Study, Behaviour Research and Therapy (2016), doi: 10.1016/j.brat.2016.01.002.

This is a PDF file of an unedited manuscript that has been accepted for publication. As a service to our customers we are providing this early version of the manuscript. The manuscript will undergo copyediting, typesetting, and review of the resulting proof before it is published in its final form. Please note that during the production process errors may be discovered which could affect the content, and all legal disclaimers that apply to the journal pertain. 


\title{
Is Metacognition a Causal Moderator of the Relationship Between Catastrophic
}

\section{Misinterpretation and Health Anxiety? A Prospective Study.}

\author{
Robin Bailey a,b, Adrian Wells a,
}

a. Division of Clinical Psychology, University of Manchester, Rawnsley Building, Manchester Royal Infirmary, Oxford Road, Manchester, M13 9WL, UK.

b.School of Health, BB235, University of Central Lancashire, Preston, Lancashire, UK, PR1 2HE.

\begin{abstract}
Psychological theories have identified a range of variables contributing to health anxiety, including, dysfunctional illness beliefs, catastrophic misinterpretation, somatosensory amplification and neuroticism. More recently, metacognitive beliefs have been proposed as important in health anxiety. This study aimed to test the potential causal role of metacognitive beliefs in health anxiety. A prospective design was employed and participants $(n=105)$ completed a battery of questionnaire at two time points (6 months apart). Results demonstrated that cognitive, personality and metacognitive variables were bi-variate prospective correlates of health anxiety. Hierarchical regression analysis revealed that only metacognitive beliefs emerged as independent and significant prospective predictors of health anxiety. Moderation analysis demonstrated that metacognitive beliefs prospectively moderated the relationship between catastrophic misinterpretation and health anxiety. Follow-up regression analysis incorporating the interaction term (metacognition $\mathrm{x}$ misinterpretation) showed that the term explained additional variance in health anxiety. The
\end{abstract}


results confirm that metacognition is a predictor of health anxiety and it is more substantive than misinterpretations of symptoms, somatosensory amplification, neuroticism, and illness beliefs. These results may have major implications for current cognitive models and for the treatment of health anxiety.

Keywords: health anxiety; metacognition; dysfunctional beliefs; catastrophic misinterpretation neuroticism; somatosensory amplification; prospective.

\section{Introduction}

Health anxiety is a condition which is characterised by beliefs that one has or will develop a serious illness based upon misinterpreting bodily symptoms (Abramowitz, Olatunji, \& Deacon, 2007; Taylor \& Asmundson, 2004). Typically considered a dimensional rather than a categorical construct (Longley et al., 2010), anxiety about one's health can range from mild transient concerns (Looper \& Kirmayer, 2001) to severe health anxiety/ hypochondriasis. Health anxiety causes high levels of psychological distress, functional impairment and excessive use of medical services (Creed \& Barsky, 2004; Lee, Creed, Ma, \& Leung, 2015). Cognitive behavioural theories consider dysfunctional beliefs to be a central cause of health anxiety (Marcus, Gurley, Marchi, \& Bauer, 2007; Norris \& Marcus, 2014). Extensive research has identified that health anxious individuals hold dysfunctional illness related beliefs (Hitchcock \& Mathews, 1992; Marcus, 1999; Marcus \& Church, 2003; Rief, Hiller, \& Magraf, 1998) and they increase health anxious symptomatology, once they are activated by health related internal or external triggers (Abramowitz \& Braddock, 2008; 
Taylor \& Asmundson, 2004). Salkovskis's cognitive model of health anxiety (Salkovskis \& Warwick, 1986; Warwick \& Salkovskis, 1990) proposes that health anxiety is linked to four specific health related dysfunctional beliefs: 'the likelihood of contracting or having an illness', ' the awfulness of illness', ' the inability to cope with illness', and 'the inadequacy of medical services for treating illness' (Salkovskis \& Warwick, 2001). A number of cross sectional studies have demonstrated that these dysfunctional beliefs are specific to health anxiety and strongly associated and predictive of this disorder (e.g. Hadjistavropoulos et al., 2012; Fergus, 2014).

In addition to dysfunctional beliefs cognitive models of health anxiety view biased interpretations in the form of catastrophic misinterpretations of bodily symptoms as important in causing health anxiety. Once dysfunctional beliefs become activated this is thought to lead the individual to catastrophically misinterpret symptoms as an indicator of the presence of potentially serious illness (Marcus \& Church, 2003; Taylor \& Asmundson, 2004). Consistent with this proposal studies have demonstrated positive associations between catastrophic misinterpretations and the presence of health anxiety (e.g. Marcus, Gurley, Marchi, \& Bauer, 2007; Norris \& Marcus, 2014; Rief, Hiller \& Margraf, 1998; Weck, Neng, Richberg, \& Stangier, 2012).

Similar to cognitive behavioural models, cognitive perceptual theories suggest that somatosensory amplification is instrumental in the development of health anxiety. The theory states that individuals with health anxiety have a tendency to be hypervigilant and selectively attend to somatic feelings and sensations in the body and consequently catastrophize these symptoms as an indicator of serious illness (Barsky, 1992; Barsky, Coeytaux, Sarnie, \& Cleary, 1993). A number of studies have identified an association between somatosensory amplification and health anxiety (Barsky \& Wyshak, 1990; Barsky, Wyshak, \& Klerman, 
1990), however greater evidence exists for the cognitive misinterpretation component of the model than heightened sensitivity to somatic symptoms (Marcus et al.,2014; Norris \& Marcus 2014).

Another important variable which has been both theoretically and clinically implicated in health anxiety is the personality construct of neuroticism. Studies have shown neuroticism to be strongly positively associated with health anxiety (McClure \& Lilienfeld, 2001; Noyes et al., 1994; 2003). In interpersonal models of health anxiety, neuroticism has been shown to be a predictor of health anxiety and strongly associated with attachment styles (Noyes, 2003). Some authors have proposed that health anxiety may just be a manifestation of neuroticism (Watson, 2012). This view however, has been challenged with studies showing that although health anxiety is associated with neuroticism it has incremental validity over this construct (Fergus \& Valentiner, 2011; Ferguson et al, 2013).

Recent advances have applied the metacognitive model of psychological disorder (Wells, 2000; Wells and Matthews, 1994; 1996) to understanding health anxiety. Central to this model is the idea that disorders such as health anxiety result from repetitive and difficult to control negative thinking marked by worry and rumination and the use of paradoxical mental control strategies such as seeking reassurance and thought suppression. Such repetitive negative thinking is the result of unhelpful metacognitions, that is underlying beliefs about thoughts, (e.g. "Thinking the worst about symptoms will keep me safe;" and "I cannot control my health worries"). The metacognitive model presents an explanation of health anxiety that is different from cognitive models, since health anxiety is seen as resulting more from extended and repetitive negative thinking about illness rather than from the belief that one is ill. Thus, according to the metacognitive model, metacognitive beliefs about repetitive thinking should be more important than beliefs about illness. This translates into 
different types of clinical practice; for example in traditional CBT a patient with health anxiety who believes that the sore throat they are experiencing is throat cancer, would be asked to generate alternative benign explanations for this symptom as a means of challenging their catastrophic misinterpretation. In contrast, in metacognitive therapy (MCT) the therapist would explore with the patient new ways of relating to the misinterpretation that consist of reduced thinking that can modify beliefs concerning the uncontrollability of healthrelated worrying. This would consist of postponing any attempt to deal with the interpretation until later. In CBT, patients are taught to challenge disease conviction and generate alternative content, however in MCT patients learn how to reduce their over-thinking response to disease convictions when they occur.

Consistent with this model several cross sectional studies have demonstrated a strong association between metacognition and health anxiety. In one study Kaur, Butow, \& Thewes, (2011) found that metacognitive beliefs were associated with an attentional bias towards positive and negative health related information (range of correlations .32 to .69). Bailey \& Wells (2013) found that metacognitive beliefs explained variance in health anxiety symptoms over and above other established correlates namely, illness cognition, somatosensory amplification, and neuroticism (range of correlations .46 to .47). Furthermore, metacognitive beliefs moderated the relationship between catastrophic misinterpretation of bodily symptoms and health anxiety (Bailey \& Wells, 2015a) calling into question the importance given to misinterpretations. In this study the moderator effect showed that catastrophic misinterpretations alone did not predict health anxiety and an input from metacognition appears to be required to produce this association. This challenges the dominant role given to catastrophic misinterpretation's as they appear inert without the 
involvement of metacognition. In an Italian community sample, Melli, Carraresi, Poli and Bailey (2016) identified metacognitive beliefs were associated with symptoms of health anxiety (range of correlations .20 to .50 ). Bailey \& Wells (In press) in a replication study found that metacognitive beliefs were again associated with health anxiety (range of correlations .52 to .68$)$

Solem et al., (2015) identified a strong relationship between health anxiety and metacognitive beliefs in a clinical sample of obsessive compulsive disorder patients (range of correlations .23 to .48 ). These earlier studies have used a range of different measures of metacognitive beliefs. For example, Melli et al (2016) used an unpublished measure, the Meta-cognitions about Heath Anxiety (MCHA), by Bouman and Meijer (1999) based on the metacognitions questionnaire (MCQ: Cartwright-Hatton \& Wells, 1997). Bailey and Wells (In Press) have used a 3 factor measure (the MCQ-HA: Bailey \& Wells, 2015b) that is more specific to health anxiety than the MCQ and is also grounded in the metacognitive model. The MCQ-HA has been shown to have incremental predictive validity over the MCQ (Bailey \& Wells, 2015b)

Although illness beliefs, catastrophic misinterpretation, somatosensory amplification, neuroticism and metacognitive beliefs are all associated with health anxiety, the majority of studies have tended to be cross-sectional in nature and therefore limit any causal interpretations. In fact when considering the amount of health anxiety research conducted to date, there are few prospective studies demonstrating temporal relationships between these variables.

In the only study of its kind, catastrophizing of bodily sensations was shown to maintain health anxiety over a one month time period (Gautreau et al., 2014). Although this is a novel finding it is limited by the brevity of the time period between measurement occasions. 
Personality factors similar to neuroticism, such as emotional stability have been shown to predict health anxiety prospectively. Ferguson (2004) found that lower levels of emotional stability positively predicted future levels of health anxiety over a 16-17 month period. Anxiety sensitivity has also been shown to be significantly associated with health anxiety Abramowitz, Deacon, \& Valentiner, 2007; Abramowitz, Olatunji, \& Deacon, 2007) in particular the "physical" dimension of this construct. However, there is only one prospective study and anxiety sensitivity did not emerge as a significant predictor (Olatunji et al, 2009)

In view of the important role assigned to metacognitive beliefs in the metacognitive model and their observed positive associations with health anxiety in cross-sectional studies, we set out to test for prospective relationships between these beliefs and subsequent health anxiety. A prospective relationship would be consistent with the hypothesised causal contribution of metacognition.

Based on cross-sectional data it was hypothesised that somatosensory amplification, neuroticism, catastrophic misinterpretations, dysfunctional illness beliefs and metacognitive beliefs would be associated with health anxiety longitudinally. In line with the main aim of the study and metacognitive theory in particular, it was hypothesised that metacognitive beliefs measured at time point 1 would explain variance in health anxiety at time point 2 (six months later), when controlling for these other associated constructs.. It was further predicted that the prospective relationship between metacognition and health anxiety would be unidirectional rather than bi-directional i.e., that metacognitions will predict later health anxiety but health anxiety will not predict later metacognition scores. Finally, based on previous cross-sectional findings and metacognitive theory we tested the hypothesis that the prospective relationship between catastrophic misinterpretation and health anxiety would be 
moderated by metacognitive beliefs rather than dysfunctional beliefs about illness (schema), and this interaction would emerge as a significant additional predictor of health anxiety over time.

\section{Methods}

\subsection{Participants}

All participants were drawn from nursing cohorts as this group have been shown to experience elevated levels of health anxiety (Azuri, Ackshota, \& Vinker, 2010; Zhang et al, 2014). Using an analogue sample can be useful when studying health anxiety as it is considered to exist on a continuum (Looper \& Kirmayer, 2001) as a dimensional construct (Ferguson, 2009; Longley et al., 2010), with mild cases being associated with clinical problems (Hadjistavropoulos \& Lawrence, 2007). Equally Marcus et al., (2007), found that elevated health anxiety and health anxious beliefs were similar in both clinical and nonclinical samples. One hundred and five participants who took part at time 1, and returned matching questionnaires at Time 2 . Twenty five participants (19.2\%) completed time 1 data but did not complete time 2 data. The Time 1 questionnaire scores of those who did and did not participate at Time 2 were not significantly different. Of the 105 participants $76(72.4 \%)$ were female and $29(27.6 \%)$ male. The age of the sample ranged from 19 to 49 years $(M=26, S D=6.52)$.

\subsection{Measures}

1.2.1 The Whiteley Index (WI: Pilowsky 1967): The Whiteley index is one of the most widely used and established measures of health anxiety and hypochondriacal symptomatology. It consists of 14 items and exists in two forms, a dichotomous yes/no format and a 5-point Likert scale. The latter version was used in this study (Welch, Carleton \& Asmundson, 2009), with scores ranging from 14 (minimum) to 70 (maximum). A number 
of studies have established that a cut off score of 40 or above indicates the presence of hypochondriasis (Gerdes et al. 1996; Noyes et al., 1994). The measure has shown good internal consistency in medical outpatients $\alpha=.80$, general practice $\alpha=.78$ and the general population $\alpha=.76$ (Speckens, Spinhoven, Sloekers, Bolk, \& van Hemert, 1996). The measure has good psychometric properties including retest reliability ( $r=.81)$ (Pilowsky, 1967), and concurrent validity (Hiller, Rief, \& Fichter, 2002; Speckens et al., 1996).

\subsubsection{Neuroticism Scale of the Eysenck Personality Questionnaire-Revised: Short}

\section{Form (EPQR-N).}

The EPQ-R short scale (Eysenck, Eysenck, \& Barrett, 1985) is a trait measure, which captures important aspects of emotional instability and anxiety (e.g., Gershuny \& Sher, 1998; Kendler, Gardner, \& Prescott, 2002). Consisting of 4 subscales: extraversion (12 items), neuroticism (12 items), psychoticism (12 items), and lie (12 items), only the neuroticism subscale was used in this study. The scale has a dichotomous "yes" or "no" format and items scored 1 or 0 , with a maximum potential score of 12 and a minimum of 0 . The neuroticism subscale has been reported to have good internal consistency with Cronbach alphas of .80 (female) and .84 (male) (Eysenck et al., 1985).

\subsubsection{The Cognitions about Body and Health Questionnaire-CABAH (Rief,} Hiller, \& Margraf, 1998). The CABAH is a 31 item self-report measure which assesses specific types of cognitions that have been shown to maintain health anxiety symptoms (Rief et al., 1998). The response format of the CABAH is a 4-point Likert scale (ranging from $0=$ "completely wrong" to $3=$ "completely right") and consists of five subscales: (1) catastrophizing interpretation of bodily complaints, (2) autonomic sensations, (3) bodily weakness, (4) intolerance of bodily complaints, and (5) health habits. Only the 
'catastrophizing interpretation of bodily complaints' (e.g. "red blotches on the skin are a threatening sign of skin cancer"); was used for this study and has been shown to have good internal consistency $\alpha=0.88$. (Rief et al., 1998).

1.2.4 Somatosensory Amplification Scale (SSAS) (Barsky, Wyshak, \& Klerman, 1990) is a ten item questionnaire which measures cognitive perceptual variables associated with health anxious/hypochondriacal symptoms: heightened sensitivity towards unpleasant bodily symptoms, selective attention towards bodily sensations, and catastrophic misinterpretations of bodily symptoms/sensations. (Barsky, Goodson, Lane, \& Cleary, 1988). Somatosensory amplification has been shown to be positively associated with hypochondriasis (Barsky et al., 1990; Marcus, Gurley, Marchi, \& Bauer, 2007) and has construct validity with measures of health anxiety such as the Whiteley Index (Barsky et al., 1990) and illness attitude scale (IAS; Kellner, 1981). The SSAS has demonstrated good internal consistency, $\alpha=.82$ and retest reliability $(r=.79)$ (Barsky et al., 1990).

\subsubsection{The Health Cognitions Questionnaire (HCQ; Hadjstavropoulos et al.,}

2012). The HCQ is a 20 item questionnaire which measures specific health anxiety related dysfunctional beliefs based upon Salkovskis \& Warwick's (2001) cognitive conceptualisation of health anxiety. Measured on a five-point scale that ranges from 1 (strongly disagree) to 5 (strongly agree), the HCQ contains the following subscales: 'Likelihood of contracting or having an illness' (HCQ-L), 'Awfulness of illness' (HCQ-A), 'Inability to cope with illness' (HCQ-C), and 'Inadequacy of medical services for treating illness' (HCQ-M). There are two separate HCQ measures one for those who have not been diagnosed with a medical illness and one for those that have; the current study used the former. The scale has shown good internal consistency, predictive and discriminative validity (Hadjstavropoulos et al., 2012). 


\subsubsection{The Meta-Cognitions about Health Questionnaire (MCQ-HA): (Bailey \&}

Wells, 2015b). This self-report measure is based on the widely used general metacognitive belief measure, the Metacognition Questionnaire (MCQ: Cartwright-Hatton \& Wells, 1997), but specifically adapted to measure health anxiety related metacognitive beliefs. The measure consists of 14 items with 4-point Likert response scales from 1 ("do not agree") to 4 ("agree very much"). Initial exploratory factor analysis (Bailey \& Wells, 2015c) has revealed a three factor structure, consisting of the following subscales: 'Beliefs that Thoughts cause Illness' (MCQ-HAC; alpha = .78), e.g., 'Thinking negatively can increase my chances of disease'; 'Beliefs about Biased Thinking' (MCQ-HAB; alpha = .83), e.g., 'I will be punished for thinking I am in good health'; and 'Beliefs that Thoughts are Uncontrollable' (MCQ-HAU; alpha $=.81$ ), e.g., 'I have no control over thinking about my health'. The factor structure was also supported through confirmatory factor analysis and the measure demonstrated discriminant, and convergent validity. The MCQ-HA also explained additional variance (24\%) in health anxiety, when controlling for the MCQ-30, thus demonstrating incremental validity.

\section{Procedure}

The study received full ethical approval from the University of Manchester's ethics committee. A convenience sample of students from a range of nursing cohorts was used. Participants were informed of the study through University email and presented with a participant information sheet outlining the study and that data would be collected over two time points. Participants at time point 1 were asked to supply an identification code known only by them which was written on the cover page of the instrument battery. When the participants consented to take part at time point 2 (six months later), they were asked to provide the same identification code as completed at time 1, so questionnaire batteries could 
be matched. Questionnaires at time 2 were administered in a randomized order in order to prevent sequencing effects.

\subsection{Data Analysis}

Pearson inter-correlations were initially run between the study variables at Time 1 and Time 2, as well as prospective correlations between Time 1 variables and the outcome (Whiteley index) at time 2. Hierarchical regression analysis was then conducted, to test for the independent prospective predictors of health anxiety at time 2 . In this approach we controlled for health anxiety at time 1 and other predictors that have been associated with the development of health anxiety, namely; neuroticism, somatosensory amplification, catastrophic misinterpretation, and dysfunctional illness beliefs and then we tested the contribution of metacognition when entered on the final steps of the model. Next we completed a moderation analysis to explore a prospective moderation model with a single moderator of the effect of X (catastrophic misinterpretation-time 1) on Y (health anxiety-time 2) by $\mathrm{M}$ (metacognition-time 1). A final hierarchical regression analysis was undertaken to ascertain whether the interaction between metacognitive beliefs and catastrophic misinterpretation explained additional variance in the equation, when controlling for all other variables.

\section{Results}

\subsection{Inter-correlations}


Descriptive statistics for all study measures at both time points are presented in Table

1. In line with cognitive and metacognitive models the majority of time 1 variables were positively and significantly correlated with time 1 health anxiety, r's ranging from $.31-.61$.

The only exceptions being the metacognitive belief "thinking causes illness" and two dysfunctional beliefs "inadequacy of medical services" and "awfulness of illness" which were not significantly associated with health anxiety. All time 2 variables were positively and significantly correlated with health anxiety, r's ranging from .24 to .53 , except for the metacognitive belief "thinking causes illness" which did not emerge as a significant correlation.

Inter-correlations were also run between all-time 1 variables and time 2 health anxiety. Results showed that the majority of time 1 variables were prospectively and significantly correlated with health anxiety at time 2 . The only exception being the dysfunctional belief "inadequacy of medical services" which was not significant.

Table 1: Descriptive statistics for study measures according to time point

\begin{tabular}{|l|l|l|l|l|}
\hline Measures & $\begin{array}{l}\text { Mean } \\
\text { Time 1 }\end{array}$ & SD Time 1 & $\begin{array}{l}\text { Mean } \\
\text { Time 2 }\end{array}$ & SD Time 2 \\
\hline MCQHAT & 17.76 & 5.14 & 18.93 & 6.07 \\
MCQHAC & 7.75 & 3.54 & 8.13 & 3.80 \\
MCQHAB & 5.87 & 1.57 & 6.10 & 1.87 \\
MCQHAU & 5.51 & 1.74 & 6.19 & 2.28 \\
CABAHT & 8.97 & 6.06 & 7.98 & 6.10 \\
WI & 26.36 & 9.36 & 22.74 & 8.27 \\
SAS & 11.93 & 5.23 & 11.61 & 5.84 \\
EPQ & 6.12 & 3.49 & 6.10 & 3.54 \\
HCQT & 56.69 & 8.52 & 56.57 & 9.86 \\
HCQC & 20.76 & 4.15 & 20.81 & 5.35 \\
HCQM & 11.65 & 1.70 & 10.59 & 2.78 \\
HCQL & 11.11 & 2.83 & 10.80 & 3.30 \\
HCQA & 14.45 & 2.57 & 14.36 & 2.89 \\
& & & & \\
\hline
\end{tabular}


MCQHAT (Metacognitions about health anxiety Total); MCQHAC (Thoughts cause illness); MCQHAB (Beliefs about biased thinking); MCQHAU (Uncontrollability of thoughts); CABAHT, (Interpretation of bodily symptoms subscale: Cognition about Body and Health Questionnaire); WI, (Whiteley Index); SAS (Somatosensory Amplification Scale); EPQ, (Eysenck Personality Questionnaire-Revised - Neuroticism Scale); HCQT (Health Cognitions Total); HCQC (Coping with Illness); HCQM (Inadequacy of medical services); HCQL (Likelihood of illness); HCQA (Awfulness of illness).

\subsection{Prospective Predictors of Health Anxiety:}

The main analysis (Table 2) tested the independent prospective predictors of health anxiety at time 2. Multicollinearity was tested by examination of variance inflation factor (VIF) and tolerance statistics. After all predictors were entered, none appeared problematic, all tolerance values were above the recommended .2 (range .38-.91; Menard, 1995) and all VIF values well less than 10 (Cohen, Cohen, West, \& Deacon, 2003; Myers, 1990).

Using time 2 health anxiety (WI2) as the dependent variable, the WI1 was entered on step one to control for time 1 health anxiety and it explained $52 \%$ of the variance $(\mathrm{p}<.001)$. EPQ-R was entered on step two to control for neuroticism, this explained $0.1 \%$ of the variance and was non-significant. Step 3 controlled for cognitive variables, catastrophic misinterpretation and somatosensory amplification and this block explained $1 \%$ of the variance and was non-significant. Dysfunctional illness beliefs were entered at step 4 and contributed $2.7 \%$ of the variance, but this was also non-significant. Finally, on step 5 the three metacognitive variables were entered as a block, and they explained $14 \%$ of the variance which was significant $(\mathrm{p}<.001)$.

In the final overall equation three variables prospectively predicted health anxiety and made a unique and statistically significant contribution to symptoms at time 2 . As expected 
health anxiety at Time 1 emerged as the strongest predictor of health anxiety $(\beta=.45, \mathrm{p}<$ $.001)$, the other two predictors were metacognitive variables; beliefs that thoughts are uncontrollable $(\beta=.27, \mathrm{p}<.005)$ and beliefs about biased thinking $(\beta=.24, \mathrm{p}<.005)$. Overall these findings show that specific health anxiety related metacognitions uniquely explained variance in health anxiety symptoms over time and their contribution was not explained by shared variance with illness beliefs, misinterpretations, neuroticism and somatosensory amplification. Indeed, these latter variables failed to emerge as prospective predictors of health anxiety at each step or in the final model.

Although the regression revealed that metacognitive beliefs were positive predictors of health anxiety, which is consistent with a causal relationship between metacognition and the development of health anxiety, it does not rule out the possibility of reciprocal causation i.e. that health anxiety may also cause elevated dysfunctional metacognitions. To test this a further regression analysis was run. The dependent variable this time was metacognitive beliefs (MCQ-HA total) at time2. On step 1 we controlled for metacognitive beliefs at time 1 (MCQ-HA total) and this explained $43 \%$ of the variance in metacognitive beliefs at time 2, and was significant. On step two we entered WI at time 1 which explained an additional 0.9\% of the variance in metacognitive beliefs at time 2 but was not significant. Time 1 metacognitive beliefs emerged as the only significant predictor $(\beta=.58, \mathrm{p}<.001)$. Overall these findings indicate that metacognitive beliefs prospectively predict health anxiety and variation in metacognition is not the consequence of health anxiety.

Table 2: Summary of hierarchical analysis predicting Time 2 health anxiety 


\begin{tabular}{|c|c|c|c|c|c|c|}
\hline \multicolumn{4}{|c|}{ STEPWISE STATISTICS } & \multicolumn{3}{|c|}{ FINAL STATISTICS } \\
\hline Step & Variable & $\Delta \mathbf{r}^{2}$ & $p$ & $\boldsymbol{\beta}$ & $\mathbf{t}$ & $p$ \\
\hline 1 (Enter) & WITI & .521 & .000 & 449 & 4.820 & .000 \\
\hline 2 (Enter) & EPQT1 & .001 & 645 & .040 & .596 & .559 \\
\hline \multirow[t]{2}{*}{3 (Enter) } & CABAHT1 & .011 & .313 & .001 & .018 & 986 \\
\hline & SAST1 & & & -.019 & -.251 & .802 \\
\hline \multirow[t]{4}{*}{4 (Enter) } & HCQ-LT1 & .027 & .226 & .028 & .413 & .680 \\
\hline & HCQ-MT1 & & & .054 & .896 & .373 \\
\hline & HCQ-AT1 & & & .124 & 1.931 & 057 \\
\hline & HCQ-CT1 & & & .015 & .240 & .811 \\
\hline \multirow[t]{6}{*}{ 5(Enter) } & MCQ- & 138 & .000 & .273 & 3.032 & .003 \\
\hline & MCQ- & & & .237 & 3.016 & .003 \\
\hline & HABT1 & & & & & \\
\hline & Dy & & & & & \\
\hline & MCQ- & & & .015 & .240 & .811 \\
\hline & HACT1 & & & & & \\
\hline
\end{tabular}

WIT1, (Whiteley Index); EPQT1, (Eysenck Personality Questionnaire-Revised - Neuroticism Scale); CABAHT1, (Interpretation of bodily symptoms subscale: Cognition about Body and Health Questionnaire); SAST1 (Somatosensory Amplification Scale);HCQ-LT1 (Likelihood 
of illness); HCQ-MT1 (Inadequacy of medical services); HCQ-AT1 (Awfulness of illness) ; HCQ-CT1 (Coping with Illness); MCQ-HAUT1 ( Uncontrollability of thoughts); MCQHABT1 (Beliefs about biased thinking); MCQ-HACT1 (Thoughts cause illness).

\subsection{Prospective Moderator Analysis}

To explore whether metacognitive beliefs at time 1 prospectively moderated the relationship between catastrophic misinterpretation and health anxiety, a moderation model was tested with a moderator of the effect of $X$ (catastrophic misinterpretation-time 1:

CABAH1) on Y (health anxiety-time 2: WIT2) by M (metacognition-time 1: MCQ-HA Total1). Using Process (command model-1), a computational bootstrapping tool for path analysis based moderation (Hayes, 2012), the moderator effect was highly significant $\mathrm{B}=$ $0.0646,95 \%$ CI $[0.02,0.10], \mathrm{t}=3.13, \mathrm{p}<.005$.

Exploration of the conditional effect of $\mathrm{X}$ on $\mathrm{Y}$ at values of the moderator, revealed the following:

1. When MCQ-HA total T1 is low there is a non-significant negative relationship between catastrophic misinterpretation $\mathrm{T} 1$ and health anxiety $\mathrm{T} 2 . B=-$ $0.1566,95 \%$ CI $[-0.4299,0.1167], t=-1.1368, p=.2584$. 
2. At the mean value of MCQ-HA total T1 there is a non-significant positive relationship between catastrophic misinterpretation $\mathrm{T} 1$ and health anxiety $\mathrm{T} 2$. $B=0.1765,95 \%$ CI $[-0.0734,0.4265], t=1.4016, p=.1642$.

3. When MCQ-HA total T1 is high there is a strong significant positive relationship between catastrophic misinterpretation $\mathrm{T} 1$ and health anxiety $\mathrm{T} 2$. $B=0.5096,95 \%$ CI $[0.1350,0.8843], t=2.699, p=.0082$.

The simple slopes analysis (Figure 1) depicts these interaction effects. It is only when MCQ-HA total is high that the prospective relationship between catastrophic misinterpretation and health anxiety strengthens and becomes significant. In essence this prospective moderation means that in order for misinterpretation to cause subsequent health anxiety, metacognitive beliefs must be highly elevated at the same time.

Of the individual subscales of the MCQ-HA, only "beliefs about uncontrollability" emerged as a significant prospective moderator, $\mathrm{B}=0.1331,95 \% \mathrm{CI}[0.01,0.25], \mathrm{t}=2.12, p$ $=.035$. 
Figure 1: Simple Slopes Analysis.

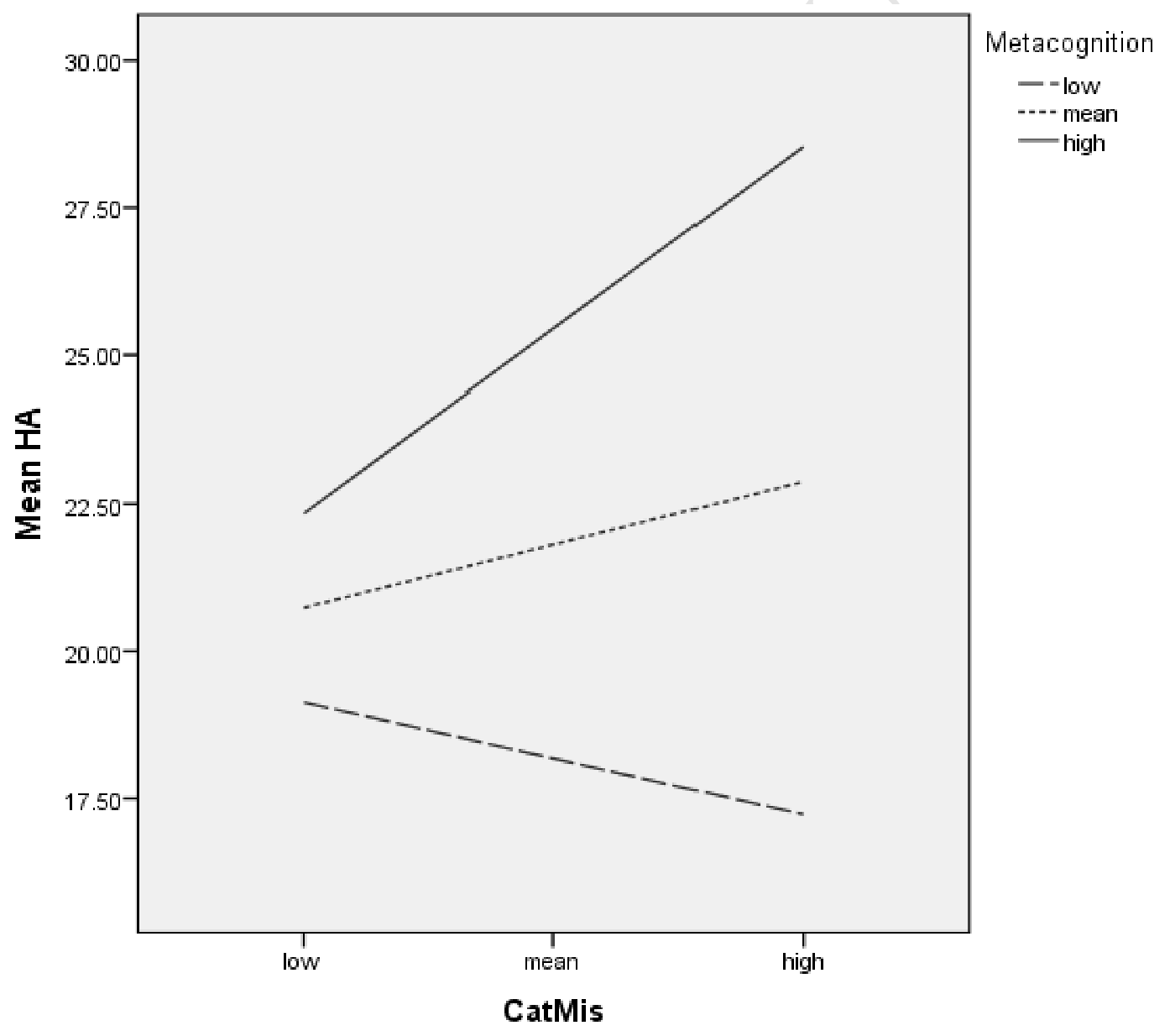


It is important to establish if the moderator effects obtained with metacognition is specific to this dimension or is a feature of other health-related (cognitive-level) beliefs. To explore this, a further model was tested with a moderator of the effect of $\mathrm{X}$ (catastrophic misinterpretation-time 1) on $\mathrm{Y}$ (health anxiety-time 2) by M (dysfunctional beliefs-time 1). The moderator effect in this instance was not significant $\mathrm{B}=0.0257,95 \% \mathrm{CI}[-0.01,0.06], \mathrm{t}$ $=1.467, p=.1453$. However the subscale "Likelihood of contracting or having an illness' (HCQ-L)" was borderline significant as a moderator $(\mathrm{B}=0.128,95 \% \mathrm{CI}[0.00,0.25], \mathrm{t}=$ $1.98, \mathrm{p}=.050$ ), suggesting that a limited range of cognitive beliefs might also impact on the relationship between misinterpretation and health anxiety. However, true moderation by such cognitions is not central to cognitive conceptualisations which typically view schemas as giving rise to misinterpretations which then lead to health anxiety. Thus, the relationship between cognition, misinterpretation and health anxiety may require further elaboration.

\subsection{Regression analysis including the interaction term.}

A further hierarchical regression analysis was undertaken to ascertain whether the interaction between metacognitive beliefs and catastrophic mis-interpretation explained additional variance in the equation, when controlling for all other variables. The same steps as the first regression analysis were employed only this time we added the interaction effect on the last step. On this occasion the interaction effect made a significant $(\mathrm{p}<.05)$ 
contribution to health anxiety at time 2 and explained an additional $2 \%$ of the variance, when controlling for the other variables.

In the final step of the equation three variables prospectively predicted health anxiety and made a unique and statistically significant contribution to symptoms at time 2 . As expected health anxiety at Time 1 emerged as the strongest predictor of health anxiety ( $\beta=$ $.41, \mathrm{p}<.001)$, the other two predictors were; beliefs that thoughts are uncontrollable $(\beta=.25$, $\mathrm{p}<.01)$ and the interaction term $(\beta=.17, \mathrm{p}<.05)$.

\section{Discussion}

This is the first study to test if metacognitive beliefs predict health anxiety six months later, when controlling for important personality variables and cognitive factors.

The study builds on earlier cross-sectional data and demonstrates several theoretically consistent bi-variate prospective correlates of health anxiety. However, the regression models show that metacognition was the only unique prospective predictor of subsequent health anxiety scores. In these analyses, cognitive and personality factors did not explain unique variance in later health anxiety. Furthermore, testing of reciprocal associations between metacognition and health anxiety across time revealed that the relationship between metacognition and health anxiety was uni-directional: metacognition appears to cause health anxiety but not vice-versa.

The two metacognition dimensions that made independent contributions were Beliefs about biased thinking (e.g. 'If I think positively about physical symptoms I will be caught off guard'), and beliefs that thoughts are uncontrollable (e.g. 'I cannot have peace of mind so 
long as I have physical symptoms'). This result is in line with the metacognitive model which predicts that beliefs about thinking are causally linked to symptoms of psychopathology, in this case health anxiety. The results are consistent with a number of other prospective studies which have demonstrated a causal relationship between metacognition, depression, and anxiety (for e.g., Hjemdal, Stiles \& Wells, 2013; Papageorgiou \& Wells 2009; Weber \& Exner 2013; Yılmaz, Gençöz, \& Wells, 2011).

Whilst the prospective associations are consistent with a causal role of metacognitions in health anxiety, these relationships could of course be a consequence of other variables that were not measured in this study. If we assume metacognitions are causal then beliefs about biased thinking may be a causal factor in health anxiety in two different ways. First, individuals may hold beliefs about the usefulness of maintaining a pessimistic stance towards symptoms as a coping strategy e.g. 'Thinking the worse about symptoms will keep me safe'. This will lead to sustained negative thinking that will maintain a sense of threat and hypervigilance for health-related information such as bodily experiences.

Second, individuals may hold beliefs regarding the danger of thinking positively and so they may actively avoid or dismiss positive health related thoughts and information, (e.g. 'Thinking positively about my health will tempt fate and I will become ill'). Similar findings were observed in an experimental study which explored the effect of health threat on attentional bias (Kaur, Butow \& Sharp, 2013). The authors found that those individuals with dysfunctional metacognitions had an attentional bias away from positive health related words after receiving reassuring feedback. In fact the association between metacognitions and attentional bias was particularly strong in the absence of objective health threat. So despite being in a positive state of mind individuals may still believe that biasing their thinking towards worry is a good thing, for example, to prevent them becoming ill. 
The metacognitive beliefs about the uncontrollability of thoughts, such as 'Only if I have a diagnosis will I be able to stop worrying' also appear to have a causal role in health anxiety. These specific beliefs are likely to be problematic as they can increase perceptions of threat regarding illness based worry and rumination, and guide attempts to control illness related thoughts, through behaviours that have counter-productive consequences. For example, trying to deal with worry by seeking reassurance transfers the control of mind to other people, and thought suppression is rarely effective leading to a persistence or greater sense of uncontrollability (Wells, 2009).

Other than metacognition the only other significant predictor of time 2 health anxiety was health anxiety at time 1 . Neuroticism, somatosensory amplification, catastrophic misinterpretation and dysfunctional beliefs all failed to emerge as unique prospective predictors. This finding stands in contrast to cross sectional data, but could indicate that these constructs are involved more in the maintenance of health anxiety rather than its cause (see Marcus et al., 2007, and Norris \& Marcus, 2014, for a review). The findings here differ from previous longitudinal studies which found that catastrophic misinterpretation (Gautreau et al., 2014) and emotional instability i.e. neuroticism (Ferguson, 2004), prospectively predicted health anxiety, but it seems that future studies must now consider metacognition in testing relationships of this kind.

In support of our final hypothesis we found that metacognitive beliefs prospectively moderated the relationship between catastrophic misinterpretation and health anxiety. Equally the interaction term explained variance in health anxiety when controlling for other variables that might explain the association, and also emerged as an independent predictor of health anxiety. This prospective data builds on earlier cross sectional data showing that catastrophic misinterpretation was only associated with health anxiety when metacognitive beliefs were 
high (Bailey \& Wells, 2015). It extends these findings by demonstrating that the moderating effects of metacognitive beliefs are protracted and can be detected 6 months later.

The regression results found in the current study are challenging for cognitive models of health anxiety which are based on a central mechanism of catastrophic misinterpretation of symptoms and associated illness related beliefs. It would seem that misinterpretations and illness beliefs are inconsequential when metacognitions are controlled. However, a nuanced picture emerges from the moderator analysis, which suggests that misinterpretations may combine with metacognition and causally predict additional variance in health anxiety. Whilst this stands in contrast to predictions of cognitive behavioural models that posit a central causal mechanism involving misinterpretations of symptoms, it does fit well with the metacognitive model of pathology where beliefs about cognition are at centre stage.

Overall if these findings are reliable they will have very important clinical implications. If metacognitive beliefs have a causal role in health anxiety targeting these beliefs may produce more long-term effects. A systematic review has revealed recovery rates from hypochondriasis to be between 30 and 50\% (olde Hartman et al., 2010). Additionally in a recent meta-analysis (Olatunji et al., 2014) on the effectiveness of CBT for health anxiety, effect sizes dramatically reduced from post treatment $(g=.95)$ to follow up $(g=.34)$. The therapeutic effects of CBT based treatments may diminish over time because treatment targets maintenance factors but may not deal with important causal factors such as metacognitions. It remains to be determined if focusing on metacognitive beliefs as a treatment priority could produce longer term benefits, enhance recovery and prevent relapse, as some preliminary data suggest (Bailey \& Wells, 2013; Papageorgiou \& Wells, 1998). 
However, the results support a closer examination of metacognition in health anxiety and a move towards evaluating metacognitive therapy (Wells, 2009) in this context.

There are a number of major limitations to the present study. The participants were predominantly female and young, so this restricts generalizability to other groups. The participants were students and therefore it is unclear whether the present findings can be generalized to the DSM-5 categories: somatic symptom disorder and illness anxiety disorder. Overall the findings of this study indicate that metacognitive beliefs might be an important variable in both the development and maintenance of health anxiety and they cast doubt over the centrality of misinterpretations and illness beliefs as causes of health anxiety.

\section{References.}

Abramowitz, J. S., \& Braddock, A. E. (2008).Psychological treatment of health anxiety and hypochondriasis: a biopsychosocial approach. Cambridge, MA, Hogrefe \& Huber Publishers.

Abramowitz, J.S., Deacon, B.J., \& Valentiner, D.P. (2007). The short health anxiety inventory in an undergraduate sample: Implications for a cognitive behavioral model of hypochondriasis. Cognitive Therapy and Research, 31, 871-883.

Abramowitz, J. S., Olatunji, B. O., \& Deacon, B. J. (2007). Health anxiety, hypochondriasis, and the anxiety disorders. Behaviour Therapy, 38(1), 86-94.

Azuri, J., Ackshota, N., \& Vinker, S. (2010). Reassuring the medical students' disease health related anxiety among medical students. Medical Teacher, 32(7), 270-275. 
Bailey, R., \& Wells, A. (2013). Does Metacognition Make a Unique Contribution to Health Anxiety When Controlling for Neuroticism, Illness Cognition, and Somatosensory Amplification? Journal of Cognitive Psychotherapy, 27(4), 327-337.

Bailey, R., \& Wells, A. (2015a). Metacognitive beliefs moderate the relationship between catastrophic misinterpretation and health anxiety. Journal of anxiety disorders, 34, 8-14.

Bailey, R., \& Wells, A. (2015b). Development and initial validation of a measure of metacognitive beliefs in health anxiety: The MCQ-HA. Psychiatry research, 230(3), 871-877.

Bailey, R., \& Wells, A. (In Press). The contribution of metacognitive beliefs and dysfunctional illness beliefs in predicting health anxiety: An evaluation of the metacognitive versus the cognitive models. Clinical Psychologist.

Barsky, A. J. (1992). Hypochondriasis and obsessive- compulsive disorder. Psychiatric Clinics and. North America, 15, 791-801

Barsky, A. J., Coeytaux, R. R., Sarnie, M. K., \& Cleary, P. D. (1993). Hypochondriacally patients' beliefs about good health. American Journal of Psychiatry, 250 (7), 1085-9.

Barsky, A. J., Goodson, J. D., Lane, R. S., \& Cleary, P. D. (1988). The amplification of somatic symptoms. Psychosomatic Medicine, 50, 510-519.

Barsky, A. J., \& Wyshak, G. (1990). Hypochondriasis and somatosensory amplification. The British Journal of Psychiatry, 157(3), 404-409.

Barsky, A. J., Wyshak, G., \& Klerman, G. L. (1990). The somatosensory amplification scale and its relationship to hypochondriasis. Journal of Psychiatry Research, 24, 323-334. 
Bouman, T. K., \& Meijer, K. J. (1999). A preliminary study of worryand metacognitions in hypochondriasis. Clinical Psychology and

Psychotherapy, 6, 96-101.

Cartwright-Hatton, S., \& Wells, A. (1997). Beliefs about worry and intrusions: The metacognitions questionnaire and its correlates. Journal of Anxiety Disorders, 11, 279-296.

Cohen J., Cohen P., West S.G., Aiken L.S. (2003). Applied multiple regression/correlation analysis for the behavioral sciences (3rd ed.). Mahwah, NJ: Erlbaum

Creed, F., \& Barsky, A. (2004). A systematic review of the epidemiology of somatisation disorder and hypochondriasis. Journal of psychosomatic research, 56(4), 391-408.

Eysenck, S. B. G., Eysenck, H. J., \& Barrett, P. (1985). A revised version of the psychoticism scale. Personality and Individual Differences, 6, 21-29.

Fergus, T. A. (2014). Health-Related Dysfunctional Beliefs and Health Anxiety: Further Evidence of Cognitive Specificity. Journal of Clinical Psychology, 70(3), 248-259.

Fergus, T. A., \& Valentiner, D. P. (2011). Intolerance of uncertainty moderates the rela-tionship between catastrophic health appraisals and health anxiety. Cognitive Therapy and Research, $35,560-565$.

Ferguson, E. (2004). Personality as a predictor of hypochondriacal concerns: Results from two longitudinal studies. Journal of psychosomatic research, 56(3), 307-312.

Ferguson, E., Ward, J. W., Skatova, A., Cassaday, H. J., Bibby, P. A., \& Lawrence, C. (2013). Health specific traits beyond the Five Factor Model, cognitive processes and trait expression: replies to Watson (2012), Matthews (2012) and Haslam, Jetten, Reynolds, and Reicher (2012). Health psychology review, 7(sup1), S85-S103. 
Ferguson, E. (2009). A taxometric analysis of health anxiety. Psychological Medicine, 39(02), 277285.

Gautreau, C. M., Sherry, S. B., Sherry, D. L., Birnie, K. A., Mackinnon, S. P., \& Stewart, S. H. (2014). Does Catastrophizing of Bodily Sensations Maintain Health-Related Anxiety? A 14Day Daily Diary Study with Longitudinal Follow-Up. Behavioural and cognitive psychotherapy, 1-11.

Gerdes, T.T., Noyes, R., Kathol, R.G., Phillips, B.M., Fisher, M., Morcuende, M., et al (1996). Physician recognition of hypochondriacal patients. General Hospital Psychiatry, 18(2), 10612.

Gershuny, B. S., \& Sher, K. J. (1998). The relation between personality and anxiety: Findings from a 3-year prospective study. Journal of Abnormal Psychology, 107, 252-262.

Hadjistavropoulos, H. D., Janzen, J. A., Kehler, M. D., Leclerc, J. A., Sharpe, D., \& BourgaultFagnou, M. D. (2012). Core cognitions related to health anxiety in self-reported medical and non-medical samples. Journal of Behavioural Medicine, 35(2), 167-178.

Hadjistavropoulos, H., \& Lawrence, B. (2007). Does anxiety about health influence eating patterns and shape-related body checking among females? Personality and individual differences, 43(2), 319-328.

Hayes, A. F. (2012). PROCESS: a versatile computational tool for observed variable medi-ation, moderation, and conditional process modeling [White paper]. Retrieved from.http://www.afhayes.com/public/process2012.pdf.

Hiller, W., Rief, W., \& Fichter, M. M. (2002). Dimensional and categorical approaches to hypochondriasis. Psychological Medicine, 32, 707-718. 
Hjemdal, O., Stiles, T., \& Wells, A. (2013). Automatic thoughts and meta-cognition as predictors of depressive or anxious symptoms: A prospective study of two trajectories. Scandinavian journal of psychology, 54(2), 59-65.

Kaur, A., Butow, P., \& Thewes, B. (2011). Do metacognitions predict attentional bias in health anxiety? Cognitive Therapy and Research, 35, 575-580.

Kaur, A., Butow, P. N., \& Sharpe, L. (2013). Health threat increases attentional bias for negative stimuli. Journal of Behavior Therapy and Experimental Psychiatry, 44,469-476.

Kendler, K. S., Gardner, C. O., \& Prescott, C. A. (2002). Toward a comprehensive developmental model for major depression in women. American Journal of Psychiatry, 159, 1133-1145.

Rief, W., Hiller, W., \& Margraf, J. (1998). Cognitive aspects of hypochondriasis and the somatization syndrome. Journal of Abnormal Psychology, 107(4), 587.

Hitchcock, P. B., \& Mathews, A. (1992). Interpretation of bodily symptoms in hypochondriasis. Behavioural Research Therapy, 30, 223-234.

Lee, S., Creed, F. H., Ma, Y. L., \& Leung, C. M. (2015). Somatic symptom burden and health anxiety in the population and their correlates. Journal of psychosomatic research, 78(1), 71 76.

Longley, S. L., Broman-Folks, J. J., Calamari, J. E., Noyes, R., Wade, M., \& Rolando, C. M.(2010). A taxometric study of hypochondriasis symptoms. Behaviour Therapy, 41(4), 505-514.

Looper, K. J., \& Kirmayer, L. J. (2001). Hypochondriacal concerns in a community population. Psychological Medicine, 31(04), 577-584.

Marcus, D. K. (1999). The cognitive-behavioral model of hypochondriasis: misinformation and triggers. Journal of Psychosomatic Research, 47, 79-91. 
Marcus, D. K., \& Church, S. E. (2003). Are dysfunctional beliefs about illness unique to hypochondriasis? Journal of Psychosomatic Research, 54, 543-547.

Marcus, D. K., Gurley, J. R., Marchi, M. M., \& Bauer, C. (2007). Cognitive and perceptual variables in hypochondriasis and health anxiety: A systematic review. Clinical Psychology Review, $27(2), 127-139$.

Menard, S. (1995). Applied logistic regression analysis. Thousand Oaks, CA: Sage.

Melli, G., Carraresi, C., Poli, A., \& Bailey, R. (2016). The role of metacognitive beliefs in health anxiety. Personality and Individual Differences, 89, 80-85.

Myers, R. (1990). Classical and modern regression with applications. Boston, MA: Duxbury.

McClure, E., \& Lilienfeld, S. O. (2001). Personality traits and health anxiety. In: Asmundson, G., Taylor, S., \& Cox, B. (Eds.), Health anxiety (pp. 65-91). New York, NY: Wiley.

Norris, A.L., \& Marcus, D. K. (2014). Cognition in Health Anxiety and Hypochondriasis: Recent Advances. Current Psychiatry Reviews, 10(1), 44-49.

Noyes, R., Jr., Kathol, R. G., Fisher, M. M., Phillips, B. M., Suelzer, M. T., \& Woodman, C. L. (1994).Psychiatric comorbidity among patients with hypochondriasis. General Hospital Psychiatry, 16, 78-87.

Noyes, R., Stuart, S. P., Langbehn, D. R., Happel, R. L., Longley, S. L., Muller, B. A., et al. (2003).Test of an interpersonal model of hypochondriasis. Psychosomatic Medicine, 65, $292-$ 300.

Olatunji, B. O., Wolitzky-Taylor. K., Elwood, L., Connolly, K., Gonzales, B., \& Armstrong, T. (2009). Anxiety sensitivity and health anxiety in a nonclinical sample: Specificity and prospective relations with clinical stress. Cognitive Therapy and Research, 33. 416-424. 
Olde Hartman, T. C., Borghuis, M.S., Lucassena, P.L.B.J., van de Laara, F.A., Speckens, A.E., \& van Weela, C. (2010). Medically unexplained symptoms, somatisation disorder and hypochondriasis: Course and prognosis. A systematic review. Journal of Psychosomatic Research, 66(5) 363-377.

Papageorgiou, C., \& Wells, A. (1998). Effects of attention training on hypochondriasis: A brief case series. Psychological Medicine, 28(01), 193-200.

Papageorgiou, C., \& Wells, A. (2009). A prospective test of the clinical metacognitive model of rumination and depression. International Journal of Cognitive Therapy, 2(2), 123-131.

Pilowsky, I. (1967). Dimension of hypochondriasis. British Journal of Psychiatry, 113, 89-93.

Salkovskis, P. M., \& Warwick, H. M. (1986). Morbid preoccupations, health anx-iety and reassurance: a cognitive-behavioural approach to hypochondriasis. Behaviour Research and Therapy, 24(5), 597-602.

Salkovskis, P. M., \& Warwick, H. C. (2001). Meaning, misinterpretations, and medicine: A cognitive-behavioral approach to understanding health anxiety and hypochondriasis. In V. Starcevic \& D. R. Lipsitt (Eds.), Hypochondriasis: Modern perspectives on an ancient malady (pp. 202-222). New York: Oxford University Press.

Solem, S., Borgejordet, S., Haseth, S., Hansen, B., Håland, Å., \& Bailey, R. (2015). Symptoms of health anxiety in obsessive-compulsive disorder: Relationship with treatment outcome and metacognition. Journal of Obsessive-Compulsive and Related Disorders, 5, 76-81.

Speckens, A. E., Spinhoven, P., Sloekers, P., Bolk, J. H., \& van Hemert, A. M. (1996).A validation study of the Whitely Index, the Illness Attitude Scales, and the Somatosensory Amplification Scale in general medical and general practice patients. Journal of Psychosomatic Research, 40(1), 95-104. 
Taylor, S., \& Asmundson, G.J.G. (2004). Treating health anxiety. A cognitive-behavioral approach. Guilford Press, New York.

Warwick, H. M. C., \& Salkovskis, P. M. (1990). Hypochondriasis. Behaviour Research and Therapy, 28(2), 105-1171.

Watson, D. (2012).Toward a comprehensive hierarchical structure. Health Psychology Review. doi: 10.1080/17437199.2011.60190.

Weber, F., \& Exner, C. (2013). Metacognitive Beliefs and Rumination: A Longitudinal Study. Cognitive Therapy and Research, 37(6), 1257-1261.

Weck, F., Neng, J.M.B., Richtberg, S. \& Stangier, U. (2012). Dysfunctional beliefs about symptoms and illness in patients with hypochondriasis. Psychosomatics, 53, 148-54.

Welch, P. G., Carleton, R. N., \& Asmundson, G. J. G. (2009). Measuring health anxiety: Moving past the dichotomous response option of the original Whiteley Index. Journal of Anxiety Disorders, 23, 1002-1007.

Wells, A. (2000). Emotional disorders and metacognition: Innovative cognitive therapy. Chichester, UK: Wiley.

Wells, A. (2009). Metacognitive therapy for anxiety and depression. New York: Guilford Press.

Wells, A., \& Matthews, G. (1994). Attention and emotion: A clinical perspective. Hove, England: Lawrence Erlbaum Associates.

Wells, A., \& Matthews, G. (1996). Modelling cognition in emotional disorder: The S-REF model. Behaviour Research and Therapy, 34, 881-888. 
Yılmaz, A. E., Gençöz, T., \& Wells, A. (2011). The temporal precedence of metacognition in the development of anxiety and depression symptoms in the context of life-stress: A prospective study. Journal of Anxiety Disorders, 25(3), 389-396.

Zhang, Y., Zhao, Y., Mao, S., Li, G., \& Yuan, Y. (2014). Investigation of health anxiety and its related factors in nursing students. Neuropsychiatric Disease and Treatment, 10, 1223. 
Highlights.

- We explore prospective associations, predictors, and moderators of health anxiety.

- All variables were bi-variate prospective correlates of health anxiety.

- Only metacognitive beliefs emerged as prospective predictors of health anxiety.

- Metacognitive beliefs prospectively moderated the relationship between catastrophic misinterpretation and health anxiety.

- Metacognitive beliefs may have a causal role in health anxiety. 